\title{
Schwerpunkt: Der Vorsorgestaat im 21. Jahrhundert
}

\section{Arno von Schuckmann}

Online publiziert: 30 . November 2020

(C) Der/die Autor(en) 2020

Wohlfahrtsstaatliche Politik ist derjenige Teil der Staatstätigkeit, der darauf gerichtet ist, vor den Wechselfällen des Lebens und vor Verelendung zu schützen und/oder die Gleichheit der Lebensführungschancen zu befördern. (Schmidt und Ostheim 2007, S. 21)

Für die soziale und somit auch kulturelle und politische Teilhabe ist ein Wohlfahrtsstaat unumgänglich. Eine moderne und demokratische Gesellschaft zeichnet sich dadurch aus, dass sich alle Mitglieder auf einen grundlegenden Wertekanon berufen können, der gesellschaftliche Partizipation für alle Bürger eines Landes ermöglicht. In der Bundesrepublik Deutschland ist der Wohlfahrtsstaat in Artikel 20 Absatz 1 des Grundgesetzes abgebildet, in dem auf das Sozialstaatsprinzip rekurriert wird.

Jedoch stellt sich zwangsläufig die Frage, wie genau der Wohlfahrtsstaat ausgestaltet werden soll. Ideen für die Ausgestaltung finden sich insbesondere bei den politischen Parteien wieder, die unterschiedlicher nicht sein könnten. Sie reichen von einer umfassenden staatlichen Fürsorgepflicht, wie sie sich insbesondere bei den linken Parteien wiederfinden, über die Betonung einer eher geringeren Staatsverantwortung und dafür mehr individuelle Vorsorge durch die Bürger selbst, bis hin zu verfassungswidrigen Vorschlägen auf der rechtsextremen Seite des Parteienspektrums, die einen exklusiven Wohlfahrtsstaat für bestimmte Bevölkerungsgruppen fordern.

Es erscheint nachvollziehbar, dass in prosperierenden Phasen die Diskussionen über den Umfang und die Leistungen des Wohlfahrtsstaates eher zugunsten der liberalen und konservativen Ideen ausschlagen, wohingegen in Zeiten ökonomischer Verwerfungen oder gesellschaftlicher Krisen eher für die progressiven Ideen plädiert wird.

\footnotetext{
A. von Schuckmann $(\bowtie)$

Institut für Politikwissenschaft, NRW School of Governance, Universität Duisburg-Essen,

Lotharstraße 53, 47057 Duisburg, Deutschland

E-Mail: Arno.vonschuckmann@uni-due.de
} 
Die ersten beiden Jahrzehnte des aktuellen Jahrhunderts sind in einem besonderen Maße geprägt von großen Krisen - man denke nur an 9/11, die daraus resultierenden kriegerischen Auseinandersetzungen und Flüchtlingsbewegungen, die Weltwirtschaftskrise sowie aktuell die Corona-Pandemie oder der seit Jahren immer evidenter werdende Klimawandel. Begleitet wurden diese Krisen zudem von einem enormen durch die Digitalisierung induzierten gesellschaftlichen Wandel, der die Sicherheit der Arbeitsplätze breiter gesellschaftlicher Schichten infragestellt. Das stellt auch die Gesellschaftswissenschaften und insbesondere die Politikwissenschaft vor die große Herausforderung, sich an der Diskussion über die Ausgestaltung des Wohlfahrtsstaates in Deutschland zu beteiligen. Aus diesem Grunde widmen wir die Rubrik Focus in diesem Heft dem Thema Der Vorsorgestaat im 21. Jahrhundert.

Zunächst wird sich Manfred Mai der Frage nach möglichen Governance-Strukturen des modernen Vorsorgestaates annehmen. Anschließend erörtert Jochen Pimpertz die Grenzen des Vorsorgestaates in einer alternden Gesellschaft. Und schließlich widmen sich Peter Weingarten und Annett Steinführer der Gleichwertigkeit der Lebensverhältnisse mit einem besonderen Blick für den ländlichen Raum.

Funding Open Access funding enabled and organized by Projekt DEAL.

Open Access Dieser Artikel wird unter der Creative Commons Namensnennung 4.0 International Lizenz veröffentlicht, welche die Nutzung, Vervielfältigung, Bearbeitung, Verbreitung und Wiedergabe in jeglichem Medium und Format erlaubt, sofern Sie den/die ursprünglichen Autor(en) und die Quelle ordnungsgemäß nennen, einen Link zur Creative Commons Lizenz beifügen und angeben, ob Änderungen vorgenommen wurden.

Die in diesem Artikel enthaltenen Bilder und sonstiges Drittmaterial unterliegen ebenfalls der genannten Creative Commons Lizenz, sofern sich aus der Abbildungslegende nichts anderes ergibt. Sofern das betreffende Material nicht unter der genannten Creative Commons Lizenz steht und die betreffende Handlung nicht nach gesetzlichen Vorschriften erlaubt ist, ist für die oben aufgeführten Weiterverwendungen des Materials die Einwilligung des jeweiligen Rechteinhabers einzuholen.

Weitere Details zur Lizenz entnehmen Sie bitte der Lizenzinformation auf http://creativecommons.org/ licenses/by/4.0/deed.de.

\section{Literatur}

Schmidt, Manfred G., und Tobias Ostheim. 2007. Einführung. In Der Wohlfahrtsstaat. Eine Einführung in den historischen und internationalen Vergleich, Hrsg. Manfred G. Schmidt, et al., 21-28. Wiesbaden: VS. 